\title{
Relationship between serum lipids and markers of bone health in post-menopausal women
}

\author{
A. D. Wood ${ }^{1}$, A. D. Hardcastle ${ }^{1}$, G. G. Duthie ${ }^{2}$, W. D. Fraser ${ }^{3}$, D. M. Reid ${ }^{1}$ and H. M. Macdonald \\ ${ }^{1}$ Bone and Musculoskeletal Research Programme, School of Medicine and Dentistry, University of Aberdeen, Foresterhill, \\ Aberdeen AB25 2ZD, UK, ${ }^{2}$ Gut Health Programme, Molecular Nutrition Group, Rowett Institution of Nutrition and Health, \\ Aberdeen AB21 9SB, UK and ${ }^{3}$ Unit of Clinical Chemistry, University of Liverpool, Liverpool L69 3GA, UK
}

CVD and osteoporosis are major chronic diseases that have a profound impact on morbidity and mortality in the UK. Epidemiological studies suggest that the two diseases are linked ${ }^{(1)}$. Osteoblasts and adipocytes share a common progenitor in the bone marrow and it has been hypothesised that an atherogenic lipid profile has a negative influence on bone formation ${ }^{(2)}$. The aim of the present study was to test the relationship between serum lipids and markers of bone health (bone mineral density (BMD) and bone-turnover markers) in postmenopausal women living in north east Scotland.

A total of 272 women (mean age 59.6 (SD 2.1) years) took part in an intervention trial investigating the mechanisms associated with the effects of fruit and vegetable intake on bone health ${ }^{(3)}$. Serum lipids were determined at 1 year for a subset of the women (placebo and fruit and vegetable arms). BMD was measured at the hip (mean of right and left hip) and lumbar spine (LS) by dual X-ray absorptiometry (Lunar Prodigy; GE Medical Systems Inc. Madison, WI, USA); the bone formation marker serum N-terminal propeptide of type 1 collagen (P1NP) and the bone resportion marker serum $\beta$ C-terminal telopeptide (CTX) were measured using enzyme chemiluminescent immunoassays (Roche Diagnostics GmbH, Mannheim, Germany). Serum LDL-cholesterol (LDL-C), HDL-cholesterol (HDL-C), TAG and total cholesterol were measured using a KONE clinical chemistry analyser (Thermo Fisher Scientific Inc., Waltham, MA, USA).

There were no significant differences in serum lipid or BMD measurements between the placebo and fruit and vegetable group. From bivariate correlations for the combined group ( $n$ 119), significant negative associations were observed between LDL-C and BMD (LS and hip), remaining significant after adjustment for weight and height, whereas the negative association between HDL-C and mean hip BMD was no longer significant after adjustment for these confounders ( $\mathrm{r}$ values shown in Table). For the bone-turnover markers, HDL-C was positively associated with P1NP at baseline and 1 year, remaining significant after adjustment for height and weight, whereas associations between HDL-C and CTX were not significant after adjustment for confounders. Serum TAG were negatively associated with P1NP and CTX at 1 year only.

\begin{tabular}{|c|c|c|c|c|c|c|}
\hline \multirow[b]{2}{*}{ Serum lipid } & \multicolumn{2}{|c|}{ Baseline BMD $\left(\mathrm{g} / \mathrm{cm}^{2}\right)$} & \multicolumn{2}{|c|}{$\mathrm{P} 1 \mathrm{NP}(\mu \mathrm{g} / \mathrm{l})$} & \multicolumn{2}{|c|}{$\mathrm{CTX}(\mu \mathrm{g} / \mathrm{l})$} \\
\hline & LS (n 119) & Hip (n 117) & $\overline{\mathrm{BL}}(n$ 119) & 1 year $(n 116)$ & $\mathrm{BL}(n$ 119) & 1 year $(n 116)$ \\
\hline HDL-C (mmol/l) & -0.114 & $-0.186^{*}$ & $0.262^{* *}$ & $0.381 * * *$ & $0.229^{*}$ & $0.222 *$ \\
\hline LDL-C (mmol/1) & $-0.241 * *$ & $-0.209 *$ & 0.126 & 0.103 & 0.000 & -0.068 \\
\hline TAG $(\mathrm{mmol} / \mathrm{l})$ & 0.131 & 0.065 & -0.150 & $-0.276^{* *}$ & -0.165 & $-0.235^{*}$ \\
\hline Total cholesterol (mmol/l) & $-0.204^{*}$ & $-0.235^{*}$ & 0.157 & 0.125 & 0.010 & -0.072 \\
\hline
\end{tabular}

$* P<0.05, * * P<0.01, * * * P<0.001$.

These results support findings relating serum lipids and BMD reported in other populations ${ }^{(2)}$. The relationship between HDL-C and $\mathrm{P} 1 \mathrm{NP}$ is consistent with the hypothesis that a healthy lipid profile is linked with bone formation. Further research is required to elucidate the mechanisms behind these observations.

This work was partly funded by the Food Standards Agency (N05043). Any views expressed are the authors' own.

1. Bagger YZ, Rasmussen HB, Alexandersen P et al. (2007) Osteoporos Int 18, 505-512.

2. Adami S, Braga V, Zamboni M et al. (2004) Calcif Tissue Int 74, 136-142.

3. Macdonald HM, Black AJ, Aucott L et al. (2008) Am J Clin Nutr 88, 465-474. 